\title{
Multilinguales
}

\section{Les interactions verbales dans le marché des Trois Horloges à Bab El Oued : structure et spécificités}

The verbal interactions in the Trois Horloges market in Bab El Oued: structure and particularities

$$
\text { والمواصفاعلات التجارية في سوق "الساعات الثلاث" بباب الواد: الهيكل }
$$

\section{Widad Bouarioua}

\section{OpenEdition}

\section{Journals}

Édition électronique

URL : http://journals.openedition.org/multilinguales/1125

DOI : $10.4000 /$ multilinguales. 1125

ISSN : 2335-1853

\section{Éditeur}

Université Abderrahmane Mira - Bejaia

Référence électronique

Widad Bouarioua, «Les interactions verbales dans le marché des Trois Horloges à Bab El Oued: structure et spécificités », Multilinguales [En ligne], 9 | 2018, mis en ligne le 01 juin 2018, consulté le 17 septembre 2019. URL : http://journals.openedition.org/multilinguales/1125 ; DOI : 10.4000/ multilinguales. 1125

Ce document a été généré automatiquement le 17 septembre 2019.

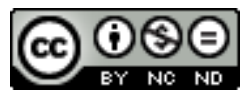

Multilinguales est mise à disposition selon les termes de la Licence Creative Commons Attribution Pas d'Utilisation Commerciale - Pas de Modification 4.0 International 


\section{Les interactions verbales dans le marché des Trois Horloges à $\mathrm{Bab} \mathrm{El}$ Oued : structure et spécificités}

The verbal interactions in the Trois Horloges market in Bab El Oued : structure and particularities

$$
\text { والتفاعلات التجارية في سوق "الساعات الثلاث" بباب الواد: الهيكل }
$$

\section{Widad Bouarioua}

1 Nous nous proposons dans cette contribution d'analyser le déroulement des interactions commerciales dans l'un des plus anciens marchés de la ville d'Alger, le marché des Trois Horloges qui se trouve dans le quartier populaire 'Bab El Oued'.

Il s'agit d'une analyse descriptive des données « authentiques et naturelles », collectées par le biais d'un magnétophone, afin de démontrer et d'expliquer l'influence des commerçants (spécialement les vendeurs algérois) sur le déroulement de ce type d'interaction verbale.

3 Notre objectif dans le présent article est d'identifier, en portant une attention minutieuse aux discours des commerçants, d'une part la structure générale des interactions commerciales ayant eu lieu dans ce site commercial. D'autre part, nous allons nous arrêter sur la particularité voire la « réalité » de la langue française dans un espace économique, social et culturel algérien, car, pour reprendre les termes de BAKHTINE, «l'interaction verbale, constitue [...] la réalité fondamentale de la langue » (1977 : 36).

Deux justifications président au choix de ce marché. Premièrement, nous avons remarqué, lors de notre enquête sur le terrain, un nombre important de vocables « nouveaux et étrangers » dont usent les commerçants algérois, en l'occurrence dans le marché des Trois Horloge. Par les vocables «nouveaux et étrangers ", nous entendons les mots qui sont soit empruntés de la langue française ou traduits des autres langues, soit inventés par ces commerçants afin d'attirer des acheteurs potentiels. 
Deuxièmement, l'existence de ces mots dans le discours des commerçants et la signification qu'ils ont nous semblent intéressantes à étudier. Nous voudrions ainsi montrer, au terme de cet article, que les commerçants dans le marché des Trois Horloges à Bab El Oued utilisent des moyens linguistiques, en exerçant un impact aussi bien sur la structure des interactions commerciales que sur le système de la langue, afin de convaincre l'acheteur.

\section{Le corpus}

6 Le corpus, qui a servi de base à cette étude, est constitué de quatre interactions commerciales dont chacune dure entre 5 et 10 minutes, il a été enregistré dans la période qui va du 3 mai au 11 juin de l'année 2015 dans le marché des Trois Horloges (Bab El Oued).

7 Il convient de préciser, ici, que ce marché contient 291 étales : 208 réservées aux légumes et aux fruits, 40 étales pour la vente des poissons et 43 pour l'alimentation générale.

8 Il ouvre tout au long de la semaine, sauf le samedi, de $8 \mathrm{~h}$ à $13 \mathrm{~h}$. Sur le plan architectural, il a une grande entrée principale et deux autres entrées secondaires. Nous tenons à faire remarquer que ce marché est visité par un nombre important de clients, entre autres, ceux qui habitent d'autres quartiers, vu l'ancienneté de ce marché, les prix raisonnables des produits et le nombre spectaculaire des commerçants informels qui l'entourent et qui étalent tout type de marchandise au sol (vêtements, produits de cosmétique et d'hygiène, alimentation générale, vaisselle, etc.).

9 Nous allons dans ce qui suit nous attarder d'abord sur l'organisation structurale, la séquentialité des interactions commerciales, en tenant compte des trois séquences importantes (séquence d'ouverture, le corps de l'interaction appelé aussi séquence thématique et la séquence de clôture). Ensuite, nous allons mettre l'accent sur le discours des commerçants de ce marché, à partir d'un corpus spontané et hétérogène, pour repérer les moyens linguistiques dont ils usent afin d'agir sur leur clientèle.

\section{La structure des interactions commerciales}

Pour mettre en relief la structure de ces interactions commerciales, nous allons nous focaliser sur les séquences qui la constituent. Autrement dit, sur «un bloc d'échanges reliés par un fort degré de cohérence sémantique et ou pragmatique " (ORECCHIONI, 1990 : 218).

\section{La structure d'ouverture}

\begin{tabular}{|l|l}
\hline Séquence d'ouverture 1 & Séquence d'ouverture 2
\end{tabular}




\begin{tabular}{|c|c|}
\hline Extrait 1 & Extrait2 \\
\hline A- Salam alikoum & C- Sbah alkhir, mrahba bik madame \\
\hline Salutation des musulmans & Oui kache makhasaq \\
\hline C- Salam, oui mrahba & -Bonjour. Soyez la bienvenue madame. Oui, à votre service vous \\
\hline Réponse à la salutation des & voulez quoi ? \\
\hline musulmans & A- Sbah alkhir. Y3aichaq. boita de confiture t3iche. \\
\hline Soyez la bienvenue. & Bonjour. Merci. Je veux une boite de confiture. \\
\hline Séquence d'ouverture 3 & Séquence d'ouverture 4 \\
\hline Extrait 3 & Extrait4 \\
\hline A-Salam khoya & A- Sbah alkhir \\
\hline Salutation des musulmans & Bonjour \\
\hline C-3alikom salam, oui & C-Sbah alkhir, an3am \\
\hline Salutations des musulmans. Oui & Bonjour, Oui. \\
\hline
\end{tabular}

11 À partir de ces quatre extraits, nous pouvons remarquer que la séquence d'ouverture permet en réalité de mettre les interactants $\mathbf{A}$ et $\mathbf{C}$ (acheteurs et commerçants) en relation. La séquence d'ouverture peut se limiter à une simple salutation comme c'est le cas dans les extraits 3 et 4 , comme elle peut être suivie par une formule de politesse « soyez la bienvenue », qui peut être interprétée comme une sorte d'invitation directe à la requête.

12 Nous déduisons donc que les séquences d'ouvertures des interactions commerciales, ayant eu lieu dans le marché des Trois Horloges, contiennent généralement des formules de politesse ritualisées. Ces formules précèdent systématiquement la requête, comme c'est le cas dans l'extrait 2 où la demande est faite d'une manière directe, que nous retrouverons dans le corps de l'interaction.

\section{Le corps de l'interaction}

13 Dans les interactions verbales, la fin de la séquence d'ouverture marque le commencement d'une deuxième séquence, nommée le corps de l'interaction ou «la phase thématique » selon les propos de Robert VION. C'est une phase qui débute par "un moment de transition " car elle contient un moment de clôture de la séquence d'ouverture pour aborder ainsi la séquence thématique ou le corps de l'interaction.

14 Dans cette phase, un des interactants annonce le thème de l'interaction et rentre dans "un terrain discursif» qui peut être "découpé en un nombre indéfini de séquences de longueur variable. » (TRAVERSO, $2005: 32$ ).

15 Il convient de faire remarquer que le corps de l'interaction est conçu par les interactionnistes comme étant la partie la plus longue dans le cadre de l'échange verbal, vu le nombre important de thèmes qui peuvent être abordés par les interactants dans le cadre de cette étape.

16 Ainsi, dans ce contexte Véronique TRAVERSO confirme qu'un « thème ne peut être clos, introduit, poursuivi, développé ou dévié que de façon coordonnée entre les participants » (Ibid., 
36). De ce fait, le corps de l'interaction est l'espace propice aux interactants afin d'aborder leurs thèmes de discussion et assurer de la sorte une continuité thématique.

Par ailleurs, nous savons que dans les interactions commerciales le corps de l'interaction (la séquence thématique) est plus au moins court si nous le comparons avec les autres types d'interactions verbales. En effet, les interactants ne cherchent pas le plaisir de l'échange, ils ont plutôt une finalité externe qui consiste dans la vente ou l'achat d'une marchandise.

18 La seconde partie de l'interaction (le corps), se compose d'un ensemble d'actions que nous pouvons énumérer comme suit : la demande ou la requête, suivie par un accusé de réception : "l'annonce du prix, du paiement et du rendu de monnaie» (ORECCHIONI et al., 2008 : 88.).

19 Pour illustrer ce point, nous proposons les deux extraits suivants :

Extrait 1

A- Wzanli zouj kilos batata w zidli kilo zrodia.

Pèse-moi deux kilos de pomme de terre et un kilo de carotte.

C- Hada makan?

C'est tout?

A- Oui hada makan, chhal ?

Oui, c'est tout. Ça fait combien?

$\mathrm{C}$ - vingt mille.

Extrait 2

C1- Hbibi 3andaq houbla 3la zouj ?

Mon ami, tu as la monnaie de deux cent dinars?

C2- Diss, diss wallah machaqit chriki

Dix, 100 DA. J'en doute. Je te jure mon ami.

C1- Sankouha walou?

Sinon 50 D A. Non plus?

C2- Wallah walou, rahi mayta hbibi, hada win rani nstaftah

Non plus, je te jure. C'est mort mon ami, je viens de commencer.

A-Khoya chhal les déodorant mana?

Mon frère, à combien sont ces déodorants ?

C2-Coul wahda wsoumatha, vingt, vingt-cinq, trente.

Chacun a son prix : vingt, vingt-cinq et trente.

A-A3tini 'Pour elle', c'est vingt-cinq yak ?

Donnez-moi 'Pour elle', c'est à vingt-cinq mille, n'est-ce pas ?

C2-Oui, tfadli.

Oui, tenez.

A-Tfadal « l'argent »

Tenez «l'argent »

C2- Maqanch sarf?

Pas de monnaie?

C1- Walou

Rien.

C2 - Mochkila sarf.

C'est un problème la monnaie.

Haki.

Tenez.

20 Dans le premier extrait, nous remarquons que la séquence thématique s'ouvre par une demande formulée explicitement par l'acheteur «Wzanli zouj kilos batata w zidli kilo zrodia » (Pèse-moi deux kilos de pomme de terre et un kilo de carotte. 
21 Suivie par une réponse positive de la part du commerçant, donnée implicitement sous forme d'une interrogation "hada makan?» ("C'est tout?»), pour dire au client «Oui, est- ce que vous voulez autre chose?». Cette réponse implicite sous-entend que la requête est obtenue. Le corps de l'interaction est clôturé par l'annonce du prix et le paiement.

De ce fait, la séquence thématique de cette interaction commerciale est constituée essentiellement d'échanges professionnels, linéaires et coordonnés, entre commerçant et acheteur, répartie selon les étapes citées en haut.

23 Le deuxième extrait, quant à lui, s'ouvre par un échange entre les deux commerçants (c1 et C2), dans lequel le premier commerçant se trouve en quête de monnaie. Cet échange se caractérise par l'utilisation d'un langage (code) propre à eux (les commerçants en question $\mathrm{C} 1 / \mathrm{C} 2)$ :

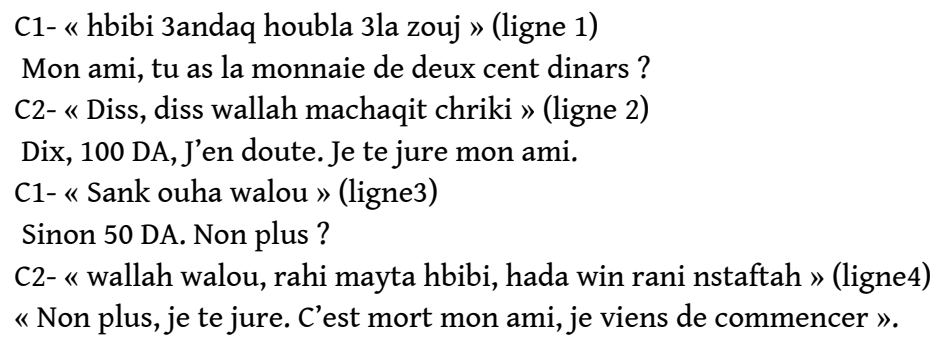

24 Nous retenons de cet échange verbal que le premier commerçant vient s'adresser au deuxième commerçant pour lui demander la monnaie d'une « houbla » ou la monnaie de deux cents dinars.

Le deuxième commerçant répond immédiatement à son interlocuteur en laissant planer le doute «Diss, diss wallah machaqit chriki » / ( Dix, 100 DA. J’en doute. Je te jure mon ami »).

Il s'agit d'une réponse négative donnée implicitement par l'emploi du vocable « machaqit/ J'en doute », renforcée par le mot « wallah » qui sert dans cet exemple à confirmer au commerçant qu'il n'est pas en train de mentir, et que ce qu'il dit est bien vrai.

27 Le deuxième commerçant enchaine dans la ligne 3 , en demandant à la place de cent dinars (100 DA), une 'senkouha' ou 50 DA. Dans la persévérance dans sa demande, nous comprenons que le deuxième commerçant se trouve réellement dans une situation de besoin, il est en quête de monnaie. La monnaie lui manque tellement qu'il envisage plusieurs alternatives.

Ce qui attire notre attention dans l'analyse des propos de ce commerçant, c'est qu'il donne la réponse dans sa question ou sa demande: en ajoutant «walou /Non plus ». C'est une sorte d'anticipation comme s'il s'attendait déjà à cette réponse, avant même de connaitre ce que son collègue allait lui dire.

La confirmation vient juste après de la part du C2 (deuxième commerçant) : "wallah walou, rahi mayta hbibi, hada win rani nstaftah » / (« Non plus, je te jure. C'est mort mon ami, je viens de commencer »).

Nous remarquons que dans cette réponse, il y a eu recours encore une fois à la connotation religieuse en utilisant le mot « wallah, », donc le vendeur jure qu'il n'a pas de monnaie.

Il confirme qu'il vient juste de commencer "hada win rani nstaftah " / "je viens de commencer ». 
Par conséquent, il n'a encore rien vendu, et donc, il n'y a pas eu encore de transactions commerciales.

C'est pourquoi la situation selon lui est décrite pas le qualificatif « mayta » ou « morte » ayant une charge sémantique négative lourde de sens.

Ce même commerçant entre après dans une interaction commerciale avec une cliente $\mathrm{A}$ qui vient pour lui demander les prix des déodorants : «A-khoya chhal les déodorants mana ?» / («Mon frère, à combien sont ces déodorants?»)

Le commerçant lui a répondu que le prix variait en fonction du produit :

C2- "coul wahda wsoumatha, vingt, vingt-cinq, trente. / / ( Chacun a son prix : vingt,

vingt-cinq et trente. »)

31 Cette proposition des prix, en fonction des produits, l'a amenée à faire son choix sans aucune hésitation en donnant le nom de son déodorant " Pour elle », peut-être préféré, parce qu'elle a tranché d'abord sur ça. "A3tini 'Pour elle', c'est vingt-cinq yak?» / (« Donnez-moi 'Pour elle', c'est à vingt-cinq mille, n'est-ce pas?»)

Ensuite, elle finit par donner elle-même son prix «Vingt-cinq yak», (« Vingt-cinq mille, n'est-ce pas?»), ce qui fait penser qu'elle a l'habitude de l'utiliser, elle connait parfaitement son prix.

L'existence du régulateur «yak» pour dire »n'est-ce pas?» a une fonction confirmative, il a été utilisé par la cliente parce qu'elle cherche à confirmer que le prix est bien « Vingt -cinq mille».

Le reste de cette séquence répond aux autres étapes que nous retrouvons dans les autres interactions commerciales, en l'occurrence l'extrait précédent, car nous repérons ce qui suit :

L'annonce du prix : qui a été faite par la cliente dans cet exemple "c'est vingt-cinq yak?» /(« vingt-cinq mille, n'est-ce pas?»).

La confirmation de ce prix vient juste après par le commerçant « oui ».

Le paiement par la cliente : «A-Tfadal » / « Tenez » (l'argent).

Rendu de monnaie par le commerçant : cette étape a été précédée par une réclamation de la monnaie, sous forme d'une interrogation directe du commerçant: C2- «maqanch sarf? » / («Pas de monnaie? ».) ( La cliente répond par un non, » Walou ».

La réponse du commerçant, en traitant l'absence de monnaie comme un vrai problème:"Mochkila sarf, c'est un vrai problème», nous permet de déduire que la réponse qu'il a déjà donnée au premier commerçant est vraie, puisque lui-même est en manque de monnaie.

Cette transaction se clôture par la restitution de la monnaie à la cliente, ce qui fait que le commerçant a pu à la fin résoudre son problème. «Donnez-moi 'Pour elle', c'est à vingt-cinq mille, n'est-ce pas?»

«Haki » / « Tenez. »

Ainsi, les séquences thématiques des interactions commerciales vues précédemment sont bien restreintes aux étapes suivantes :

La requête ou la demande de la marchandise par l'acheteur.

La réponse à la demande par le commerçant.

L'annonce du prix par le commerçant.

Le paiement par le client.

Rendu de la monnaie par le commerçant quand c'est nécessaire (obligatoire).

34 Voyons à présent un autre exemple : 
A- « chhal mana wlidou »

«Combien ça mon fils?»

C- «Cinquante mille, yama »

«Cinquante mille ma mère »

" wach ha lghla yawlidi ? ay $12 \mathrm{H}$ w mazelou sardine ghali. »

«C'est quoi cette cherté ? Il est $12 \mathrm{H}$ et les sardines sont toujours chères. »

C- « wach diri yama, aw nakas chwia had laymat »

"Que va-t-on faire ma mère ? Il y a un manque des sardines ces derniers temps. "

«Allah yarham lyamat qui kane sardine mamnou3 yk3ad hata $12 \mathrm{~h}$, limaytba3ch

yatarma. Ih ya wlidi doka chtaqnah»

« Où sont les anciens jours où les sardines ne restaient pas jusqu'à $12 \mathrm{H}$ ? Ce qui ne

se vendait pas, on le jetait s'il restait à $12 \mathrm{H}$. Maintenant, ça nous manque ».

À la différence de la première séquence thématique, nous soulignons que dans cet extrait la requête n'a pas été satisfaite. Cette insatisfaction a fait que l'échange entre l'acheteur et le commerçant a pris une autre forme de type "requête/ impossibilité de satisfaire la requête " (TRAVERSO, 2005: 58), ce qui a donné lieu à un court échange discursif entre les interactants (acheteur et commerçant) dans lequel la cliente critique l'actuelle situation économique algérienne, au sujet de l'inflation des prix. Elle exprime une sorte de nostalgie de l'époque où les prix étaient abordables, surtout ceux des sardines qui ne se vendaient pas avant $12 \mathrm{H}$, car elles finissaient parmi les détritus. Maintenant ça ne manque conclut la cliente, appelée par le commerçant par «yama/ ma mère", ce qui fait comprendre qu'elle est d'un certain âge. Elle fait une comparaison entre les prix d'avant et ceux d'aujourd'hui.

\section{La séquence de clôture}

Une séquence qui » correspond à la fermeture de la communication et à la séparation des participants " (TRAVERSO, 1996: 48). Durant cette dernière phase de l'interaction verbale, les interactants marquent la fin de leurs échanges verbaux, c'est pourquoi elle est considérée comme une partie délicate, dans la mesure où, pour reprendre ainsi les termes de LAVER, "elle a pour tâche non seulement d'organiser la fin de la rencontre, mais aussi de déterminer comment les interlocuteurs vont se quitter l'un de l'autre " (LAVER cité par ORECCHIONI, 1990 : 222).

Dans notre corpus d'étude, nous tenons à signaler que toutes les interactions commerciales que nous avons enregistrées contiennent cette séquence de clôture. C'est une phase omniprésente même quand l'interaction n'aboutit pas à la vente d'une marchandise, elle est composée généralement des remerciements et des expressions de bénédiction comme le montrent ainsi les extraits suivants :

\begin{tabular}{|l|l|}
\hline Séquence de clôture 1 & Séquence de clôture 2 \\
\hline Saha, Allah y3awnaq & A-Sahit, saha \\
Merci, que Dieu vous aide. & $\begin{array}{l}\text { Merci, que Dieu vous donne la santé } \\
\text { C-Saha, Allah ysalmaq. } \\
\text { C- Saha. }\end{array}$ \\
Merci. & Merci, que Dieu vous protège. \\
\hline Séquence de clôture 3 & Séquence de clôture 4 \\
\hline
\end{tabular}




\begin{tabular}{|l|l|}
\hline C-C'est bon, saha. & A- Saha, Allah y3awnaq. \\
C'est bon, Merci. & Merci, que Dieu vous aide. \\
A-Saha. & C-Allah yastarq, Saha. \\
Merci. & Que Dieu vous protège. Merci. \\
\hline
\end{tabular}

Si nous observons les séquences de clôture en haut, nous pouvons constater qu'elles sont caractérisées par la présence des formules de politesse euphorisantes de type "Merci, que Dieu vous aide. / Merci, que Dieu vous protège. »

Ces formules, ayant la valeur de remerciements, interviennent spontanément après la séquence de paiement. Elles annoncent le départ de l'acheteur et la séparation physique des deux interactants (commerçant et acheteur).

\section{Remerciements et rites de politesse}

Il s'agit de procédés linguistiques "pertinents » qui consistent à « respecter un certain nombre de règles et de conventions » (TRAVERSO, $2005: 53$ ).

Par le choix de ces deux procédés, les interactants visent à manifester le respect qu'ils ont les uns pour les autres. Les rites de politesse sont omniprésents dans toutes les interactions commerciales sur lesquelles nous avons travaillé, précisément dans les deux séquences d'ouverture et de clôture.

Notons que dans certains cas les remerciements peuvent avoir lieu même dans le corps de l'interaction, au moment où le commerçant rend la monnaie à son client ou quand le client se trouve satisfait suite à une réduction du prix de la part de commerçant. Nous constatons donc que la plupart de ces expressions sont des formulations votives qui sont en arabe dialectal, des fois de nature religieuse que nous pouvons même trouver en dehors des transactions commerciales.

Les remerciements et les rites de politesse utilisés par nos interactants sont répertoriés dans le tableau qui suit :

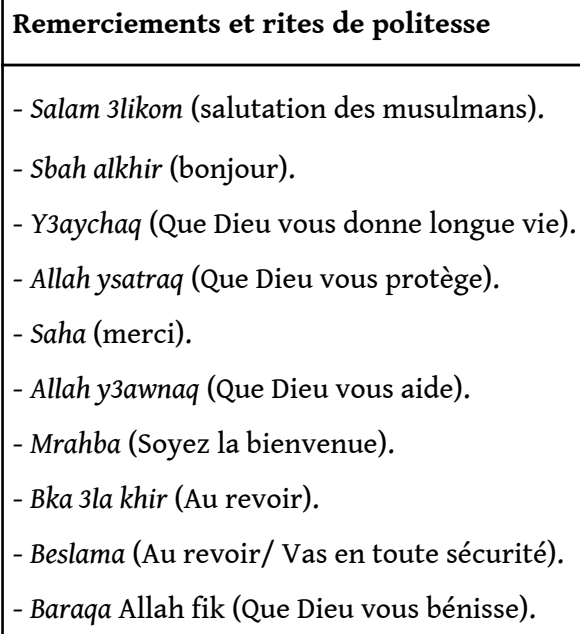




\section{Lexique spécifique des commerçants}

Les échanges entre les participants (commerçants et acheteurs) aux interactions commerciales étudiées ont une particularité qui répond bien à la situation linguistique algérienne : d'un côté, les interactions sont caractérisées par l'existence du phénomène de l'alternance codique entre l'arabe dialectal et la langue française, d'un autre côté, elles contiennent un nombre important de nouvelles unités lexicales (la créativité lexicale). Pour clarifier ce point, nous allons reprendre dans ce qui suit chaque point à part :

\section{L'alternance codique}

"Changement ou alternance de langues ou de variétés linguistiques ", " code switching » ou " code mixing» selon la terminologie anglo-saxonne sont de différentes appellations pour désigner un même phénomène linguistique qui s'installe quand

le locuteur de LX, transfère des éléments ou règles de LY, à quelque niveau linguistique que ce soit phonologique, grammatical et lexical ; ces éléments ne sont pas intégrés au système linguistique de la $\mathrm{LX}$, sinon ils seraient considérés comme emprunts (TRAVERSO, 1996 : 144)

De ce fait, nous pouvons dire qu'il s'agit d'un procédé qui consiste à utiliser alternativement dans le même énoncé deux ou plusieurs langues. GYMPERZ considère l'alternance codique comme étant « la juxtaposition à l'intérieur d'un même échange verbal de passages où le discours appartient à deux systèmes ou sous-systèmes grammaticaux différents » (1989: 57).

Pour ce qui est des échanges interactionnels des participants aux interactions commerciales étudiées, nous avons pu repérer les deux types d'alternances suivants : l'alternance codique intra-phrastique et l'alternance codique extra-phrastique.

Pour ce qui est de l'alternance codique intra-phrastique, c'est le type d'alternance le plus répandu dans nos interactions commerciales. En effet, il se caractérise par l'existence de segments appartenant à deux langues différentes.

Voici quelques exemples :

1- « Mon frère, chhal mana?»

"Mon frère, c'est à combien par-là?»

2- «Les olives noires, chhal rahoum?»

«Combien sont les olives noires?»

3- « Kayna fiha d'autres coulours mana?»

"Ii y en a dans d'autres couleurs?»

4- « La grande taille kayna mana, c'est disponible?»

«Avez-vous la grande taille? Est-elle disponible?»

5- « 3andaq déodorant?»

«Avez-vous du déodorant?»

51 Nous constatons à partir des exemples présentés ci-dessus que les interactants (précisément les acheteurs) alternent dans la même phrase deux langues. C'est un mélange de langues qui se fait d'une manière automatique et spontanée dans la mesure où il ne dérange ni le déroulement des interactions commerciales ni la compréhension mutuelle des interactants.

Par opposition au premier type, l'alternance codique extra phrastique est moins récurrente dans notre corpus. Ce constat s'explique principalement par le fait que nos 
locuteurs ne font pas recours à une autre langue pour introduire des expressions comme les remerciements (saha), les salutations (sbah alkhir, salam 3likom) et les souhaits (Allah y3awnaq).

Ils préfèrent plutôt les formuler en langue arabe dialectal car ils se sont habitués à ces formulations figées qui se trouvent ancrées dans le comportement langagier de nos informateurs. D'autant plus, certaines expressions sont à connotation religieuse telles (Salam 3alikom, Allah y3awnaq, etc. » d'où la pertinence de les dire dans leur langue d'origine.

\section{La création lexicale}

La création de nouvelles unités lexicales est un procédé linguistique d'une grande importance dont ils se servent nos interactants (commerçants). Ces unités linguistiques, confédérées comme nouvelles innovations, n'empêchent pas la compréhension mutuelle entre le commerçant et l'acheteur.

Ainsi, le tableau ci-dessous regroupe les nouvelles unités lexicales retenues :

\begin{tabular}{|l|l|}
\hline Nouvelle unité & Signification \\
\hline Houbla & 200 dinars \\
\hline Defra & 10 dinars \\
\hline Senkouha & 50 dinars \\
\hline Diss & 100 dinars \\
\hline Zarbia & L'ancien 100 dinars \\
\hline Chriki & Mon ami \\
\hline Qis-qis & 5 dinars \\
\hline Paskal & 50 francs français \\
\hline Meska Taureau & 1000 dinars \\
\hline Hadjra & 10.000 dinars. \\
\hline 'Arg & 1.000 .000 dinars \\
\hline
\end{tabular}

56 Nous ouvrons, ici, une parenthèse pour préciser que ces mots ont un rapport direct avec le domaine commercial. Ce sont des mots qui reviennent dans le discours des jeunes commerçants lors de leurs transactions commerciales (surtout quand le commerçant est un jeune de sexe masculin) dans le marché des Trois Horloges à Bab El Oued.

57 À la fin de la présente étude, et à partir de ce qui a été dit précédemment, nous insistons sur un point si important à nos yeux, et qui était d'ailleurs à l'origine de ce 
modeste travail de recherche, le terrain linguistique algérien est riche en phénomènes linguistiques qui méritent d'être analysés de près.

Porter un œil analytique sur le déroulement des interactions commerciales dans les marchés algérois et particulièrement dans le marché des Trois Horloges (Bab El Oued), nous a permis de repérer, d'abord la structure générale de ce type d'interaction qui contient toutes les étapes décrites par les spécialistes de ce domaine à savoir :

La séquence d'ouverture qui permet d'établir le contact entre le commerçant et l'acheteur, elle est formulée par des salutations ritualisées. Le corps de l'interaction qui se compose d'une suite d'actions : requête, réponse à la demande, paiement, rendu de monnaie et enfin une séquence de clôture qui annonce la séparation des deux interactants, elle contient, elle aussi, des formules de politesse et des remerciements.

La présence sur le Terrain (le marché des Trois Horloges dans notre cas) nous a permis d'être entre autres en contact direct avec la langue comme système de signes mis en pratique.

61 Cette mise en pratique de la langue par nos enquêtés nous amène à nous rendre compte de l'état de la langue française dans un contexte économique algérois, en l'occurrence le marché des Trois Horloges. Ce constat aboutit à la découverte de la dynamique langagière dans le cadre des interactions verbales et précisément les interactions commerciales algériennes et d'en saisir les spécificités et les codes linguistiques mis en œuvre.

\section{BIBLIOGRAPHIE}

ASSELAH-RAHAL, Safia, Plurilinguisme et migration, Edition Harmattan, Paris, 2004.

BABAA, Chafika, L'étude des rituels de salutations et de remerciements dans les transactions algériennes. Approche interculturelle et contrastive, Université de Constantine, 2005.

BAKHTINE, Mikhail, VOLOCHINOV, Valentin., Le marxisme et la philosophie du langage. Essai d'application de la méthode sociologique en linguistique. Édition Minuit, Paris, 1977.

BLANCHET, Philippe, La linguistique de terrain, méthodes et théories une approche ethnolinguistique, Presses Universitaires, Paris, 2000.

DARCHERIF, Zoulikha, Analyse interactionnelle dans des transactions commerciales en Algérie, l'université de Tlemcen, 2014.

DE SINGLY, François, L'enquête et ses méthodes : Le questionnaire, Edition Nathan, Paris, 1992.

DIMECHKI, Loubna, L'analyse des interactions de commerce en France et au Liban : une perspective comparative interculturelle, Université Lyon 2, Paris, 2003.

GOFFMAN, Erving, Les rites d'interaction, Edition de Minuit, Paris, 1974.

GYMPERZ, John, Sociolinguistique interactionnelle. Une approche interprétative, Edition Harmattan, Paris, 1989. 
HEDID, Souhila, Le français dans les transactions commerciales en milieu urbain. Analyse pragmatique des requêtes dans les agences de voyages à Constantine, Université de Constantine, 2005.

KEFSI, Radouane, L'analyse conversationnelle des vendeurs et des vendeuses dans les show-Room de Constantine. Etude de la négociation, Université de Constantine, 2011.

KERBRAT - ORECCHIONI, Catherine, Les interactions verbales, Tome1, Edition Armand Colin, Paris, 1990.

KERBRAT - ORECCHIONI, Catherine, La conversation, Seuil, Paris, 1996.

KERBRAT - ORECCHIONI, et al, Les interactions en site commercial. Invariants et variations, Paris, 2008.

TRAVERSO, Véronique, La conversation familière. Analyse pragmatique des interactions, Puf, Presses Universitaires, Paris, 1996.

TRAVERSO, Véronique, L'analyse des conversations, Edition Armand Colin, Paris, 2005.

TRINH, Duc Thai, Etude comparative du fonctionnement des interactions dans les petits commerces en France et au Vietnam, Université de Lyon 2, Paris, 2002.

VION, Robert, La communication verbale. Analyse des interactions, Hachette, Paris, 2000.

\section{RÉSUMÉS}

Notre travail porte sur le déroulement des interactions commerciales dans l'un des marchés de la ville d'Alger, à savoir le marché des Trois Horloges à Bab El Oued. Il s'agit d'une étude qui se veut descriptive afin de repérer la structure générale de ce type d'interaction (séquence d'ouverture, le corps de l'interaction appelé aussi séquence thématique, séquence de clôture, participants, etc.) et les spécificités du discours des commerçants de ce site commercial.

Pour ce faire, nous nous sommes basés sur quatre interactions commerciales, enregistrées dans ce marché, dans lesquelles les interactants entrent en transactions commerciales les uns avec les autres.

Il nous a été révélé à la fin de cette étude que ces interactions commerciales sont de la structure suivante : séquence d'ouverture (contenant des salutations et des formules ritualisées), le corps de l'interaction (contenant la requête, la réponse à la demande, rendu de monnaie) et la séquence de clôture qui se compose des remerciements et des vœux annonçant la séparation des participants.

Il nous a été dévoilé aussi qu'au sein de ce type d'interaction les participants, en l'occurrence les commerçants, utilisent un lexique spécifique comportant entre autres l'alternance codique (de type intra- phrastique) et de nouveaux mots grâce à la créativité lexicale.

Our work focuses on the development of commercial interactions in one of the markets Algiers, namely the Trois Horloges market in Bab El Oued. This is a descriptive study to identify the general structure of this type of interaction (opening sequence, the body of the interaction also called thematic sequence, closing sequence, participants, etc.) and the specificities of the traders' speech of this commercial site.

To reach our aim, we have based our research on four commercial interactions, recorded in this market, in which seller and the buyer enter into commercial transactions with each other.

At the end of this study we noticed that these commercial interactions are of the following structure : opening sequence (containing greetings and ritualized formulas), the body of the interaction (containing the query, the response to the request, return of money) and the closing sequence which consists of the thanks and greetings announcing the separation of the participants. 
It has also been revealed to us that within this type of interaction, the participants in this case the traders use a specific lexicon such as : the code switching (of the intra-phrastic type) and new words thanks to lexical creativity.

INDEX

Mots-clés : interaction commerciale, séquence, créativité lexicale, rite de politesse, alternance codique

التفاعلات التجارية, تسلسل, إبداع معجمي, طقوس مهذبة, تشفيرفهرس الكلمات المفتاحية:

Keywords : commercial interaction, sequence, lexical creativity, politeness rituals, code متناوب switching

\section{AUTEUR}

WIDAD BOUARIOUA

Université Alger 2 